\title{
PROPAGATION PATH AND FATIGUE LIFE PREDICTIONS OF BRANCHED CRACKS UNDER PLANE STRAIN CONDITIONS
}

\author{
M.A. Meggiolaro, A.C.O. Miranda, J.T.P. Castro, L.F. Martha \\ Pontifical Catholic University of Rio de Janeiro \\ Rio de Janeiro, RJ, Brazil 22453-900
}

\begin{abstract}
Fatigue cracks can significantly deviate from their Mode I growth direction due to the influence of overloads, multi-axial stresses, micro structural inhomogeneities such as grain boundaries and interfaces, or environmental effects, generating crack kinking or branching [1]. The stress intensity factors (SIF) associated to branched fatigue cracks can be considerably smaller than that of a straight crack with the same projected length, causing crack growth retardation or even arrest. This mechanism can quantitatively explain retardation effects even when plasticity induced crack closure cannot be applied, e.g. in high R-ratio fatigue problems under plane strain conditions. Analytical solutions have been obtained for the SIF of some branched cracks, however numerical methods are the only means to predict the subsequent curved propagation behaviour. In this work, a specialized Finite Element program is used to calculate the propagation path and associated SIF of bifurcated cracks. The numerical calculations are validated through experiments on 4340 steel ESE(T) specimens. A total of 6,250 FE calculations are used to fit empirical equations to the process zone size and crack retardation factor along the curved crack branches. The bifurcation simulations include several combinations of bifurcation angles, branch asymmetry ratios, crack growth exponents, and even considers interaction between crack branching and other retardation mechanisms such as crack closure, assuming the crack opening level is well known.
\end{abstract}

\section{Introduction}

A fatigue crack deviated from its nominal Mode I plane induces mixed-mode near-tip conditions even if the far-field stress is pure traction. For instance, a pure Mode I stress intensity factor (SIF) $K_{l}$ induces Modes I and II SIF $k_{1}$ and $k_{2}$ near the longer branch of a bifurcated crack and $k_{1}$ ' and $k_{2}$ ' near the shorter one. The equivalent SIF $K_{b}$ and $K_{c}$ of the longer and shorter branches, calculated respectively from $\left(k_{1}, k_{2}\right)$ and $\left(k_{1}, k_{2}\right)$ using e.g. the $\sigma_{\theta \max }$ criterion [2], can be considerably smaller than that of a straight crack with the same projected length, see Fig. 1. Therefore, such branching can retard or even arrest subsequent crack growth [3].

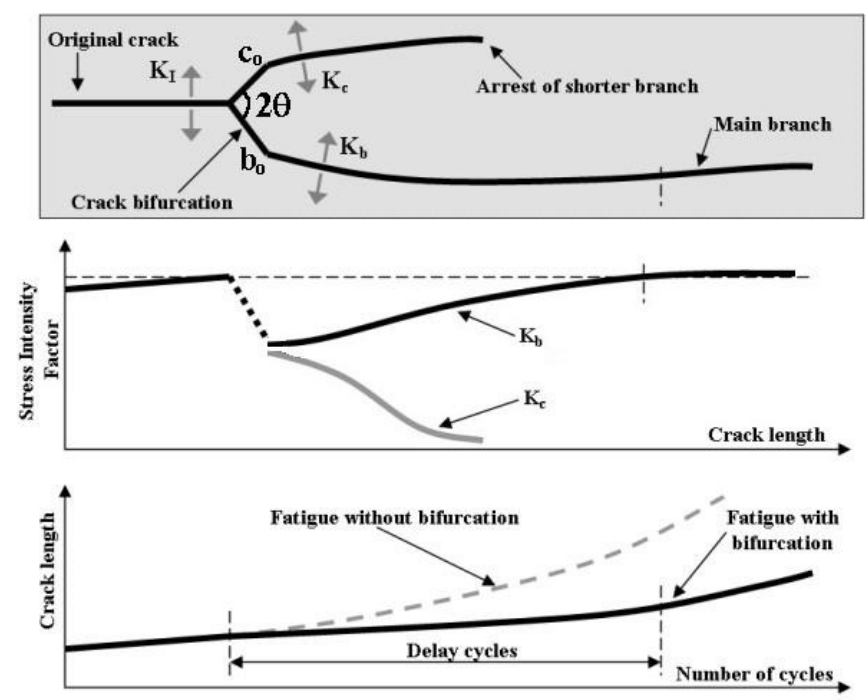

Figure 1. Bifurcated crack propagation behavior. 
It is experimentally observed that very small differences between the crack branch lengths $b$ and $c$ are enough to cause the shorter branch $c$ to arrest while the larger one $b$ propagates, generally changing its curvature at a retarded rate until returning approximately to its pre-overload SIF and growth direction and rate, see Fig. 1. Therefore, although many branches can be developed along the main crack path, only the fastest branch continues to grow, while all others are brought to a stop due to a shielding effect. This typical propagation behavior has been observed in many structural components, e.g. on a branched crack on an aircraft wheel rim made of 2014-T6 aluminum alloy [4].

Some analytical solutions have been obtained for the SIF of kinked and branched cracks, but it is generally recognized that it is very difficult to develop accurate analytical solutions to their complex propagation behavior [5-9]. Therefore, numerical methods such as Finite Elements (FE) and Boundary Elements (BE) are the only practical means to predict the propagation behavior of branched cracks [10]. A summary of such SIF solutions as a function of the deflection angle and the length of the deflected part of the crack is presented in [11].

To predict the (generally curved) path of a branched crack and to calculate the associated Modes I and II SIF, a specially developed interactive FE program named Quebra (meaning fracture in Portuguese) is used [12]. This program simulates twodimensional fracture processes based on a FE self-adaptive strategy, using appropriate crack tip elements and crack increment criteria. The adaptive FE analyses are coupled with modern and very efficient automatic remeshing schemes. An efficient meshing algorithm is fundamental to avoid elements with poor aspect ratio, since the ratio between the size of the larger and smaller elements can be above 1,000 in crack bifurcation calculations. To accomplish that, Quebra uses an innovative algorithm incorporating a quadtree procedure to develop local guidelines to generate elements with the best possible shape. The internal nodes are generated simultaneously with the elements, using the quadtree procedure only as a node-spacing function. This approach tends to give a better control over the generated mesh quality and to decrease the amount of heuristic cleaning-up procedures. Moreover, it specifically handles discontinuities in the domain or boundary of the model. Finally, to enhance the mesh element shape quality, an a posteriori local mesh improvement procedure is used [13]. The crack propagation calculations are described in the next section.

\section{Propagation of Branched Cracks}

The growth of branched cracks is studied using the Quebra program to model a $\mathrm{C}(\mathrm{T})$ specimen with width $w=32.0 \mathrm{~mm}$, crack length $a=14.9 \mathrm{~mm}$, and a very small bifurcation with angle $2 \theta$ ranging from $40^{\circ}$ to $168^{\circ}$, initial longer branch length $b_{0}=10 \mu m$ and initial shorter branch lengths ranging from $c_{0}=5 \mu \mathrm{m}$ to $10 \mu \mathrm{m}$. A fixed crack growth step of $\Delta b=3 \mu \mathrm{m}$ (or $1 \mu \mathrm{m}$ during the first propagation steps) is considered for the propagation of the longer branch $b$. This growth step is calculated in the direction defined by the $\sigma_{\theta \max }$ criterion [2]. Due to the differences in the SIF and crack growth rate, a growth step $\Delta c$ smaller than $\Delta b$ is expected for the shorter branch. This smaller step is obtained assuming a crack propagation law that models the first two growth phases,

$$
\frac{d a}{d N}=A \times\left(\Delta K-\Delta K_{t h}\right)^{m}
$$

where $A$ and $m$ are material constants and $\Delta K_{\text {th }}$ is the propagation threshold at a given $R$ ratio. If $\Delta K_{b}$ and $\Delta K_{c}$ are respectively the stress intensity ranges of the longer and shorter branches, then the growth step $\Delta c$ of the shorter branch $c$ should be

$$
\Delta c=\Delta b \cdot\left(\frac{\Delta K_{c}-\Delta K_{t h}}{\Delta K_{b}-\Delta K_{t h}}\right)^{m}
$$

Interestingly, the ratio between the propagation rates of the two branches is independent of the material constant $A$. In this analysis, the exponent $m$ is assumed to be 2.0, 3.0, and 4.0, which are representative for the range of the measured exponents for structural alloys. A similar expression can be obtained if other crack retardation mechanisms are considered, through Lang and Marci's propagation threshold $K_{P R}[14]$ with $A$ and $m$ parameters fitted for each considered load ratio $R$ :

$$
\Delta c=\Delta b \cdot\left(\frac{K_{\max , c}-K_{P R}}{K_{\max , b}-K_{P R}}\right)^{m}
$$

where $K_{\max , b}$ and $K_{\max , c}$ are the maximum SIF of the longer and shorter branches respectively. This threshold $K_{P R}$ can be interpreted as the limiting value $K_{\max }$ of the Unified Approach $[15,16]$.

Both the crack path and the associated SIF along each branch are obtained using the Quebra program. Several calculations were performed for different values of the exponent $m$, bifurcation angle $2 \theta$, relation $c_{0} / b_{0}$, and SIF, considering or not the effect of $K_{P R}$, as described next.

First, the propagation behavior of branched cracks is studied using FE but neglecting any retardation mechanism other than the bifurcation itself (i.e. assuming $K_{P R}=0$ ). Figure 2 shows the contour plots of the normal stress component in the load 
direction axis and propagation results for a bifurcated crack with angle $2 \theta=150^{\circ}$, obtained from the $\mathrm{FE}$ analysis for $c_{0} / \mathrm{b}_{0}=$ $0.91, m=2$ and no closure. In this figure, the deformations are highly amplified to better visualize the crack path. Note that the crack path deviates from the original branch angles, deflecting from $\pm 75^{\circ}$ to approximately $\pm 28^{\circ}$. In addition, the originally shorter branch arrests after propagating (only) about $29 \mu \mathrm{m}$, while the longer branch returns to the pre-overload growth direction and SIF (even though the subsequent crack growth plane may be offset from the pre-overload one, see Fig. 2).
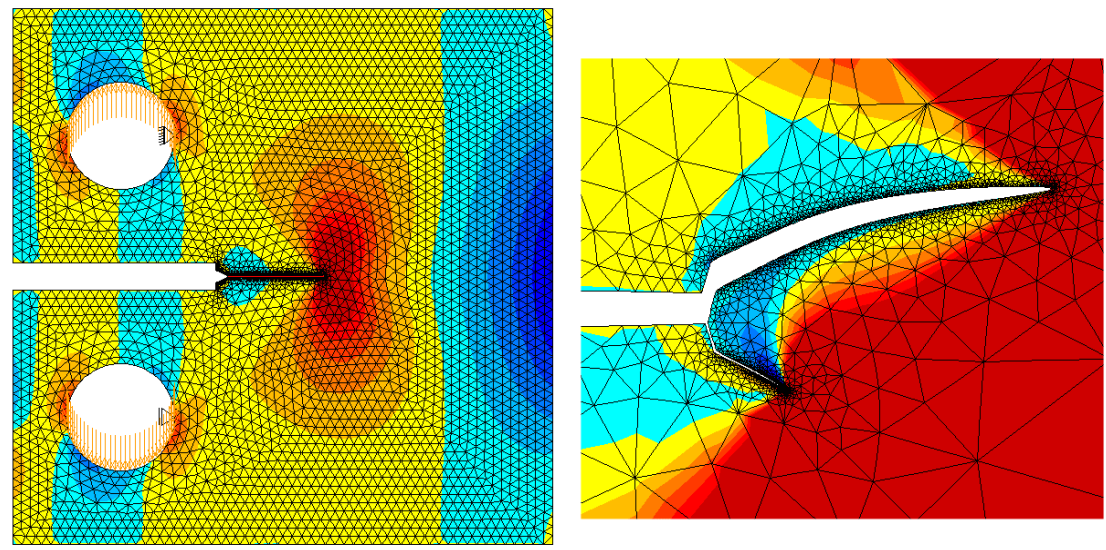

Figure 2. Propagation simulation of a bifurcated crack on a $\mathrm{C}(\mathrm{T})$ specimen (left), and close-up view of the two original $11 \mu \mathrm{m}$ and $10 \mu \mathrm{m}$ branches with angle $2 \theta=150^{\circ}$ (right).

Figure 3 shows the crack paths obtained from the FE analyses of bifurcated cracks with $2 \theta=130^{\circ}$ and $c_{0} / b_{0}=\{0.5,0.8,0.95$, $1\}$, considering $m=2$ and $K_{P R}=0$. The dashed lines show the theoretical propagation behavior of a perfectly symmetric bifurcation $\left(c_{0} / b_{0}=1\right)$. In this case, the retardation effect would never end because both branches would propagate symmetrically without arresting. Clearly, such behavior is not observed in practice, since the slightest difference between $b_{0}$ and $c_{0}$ would be sufficient to induce an asymmetrical behavior. Figure 3 also shows that lower $c_{0} / b_{0}$ ratios result in premature arrest of the shorter crack branch, leading to smaller retardation zones. Also, the propagation path of the longer branch is usually restrained to the region within the dashed lines, while the shorter one is "pushed" outside that envelope due to shielding effects.

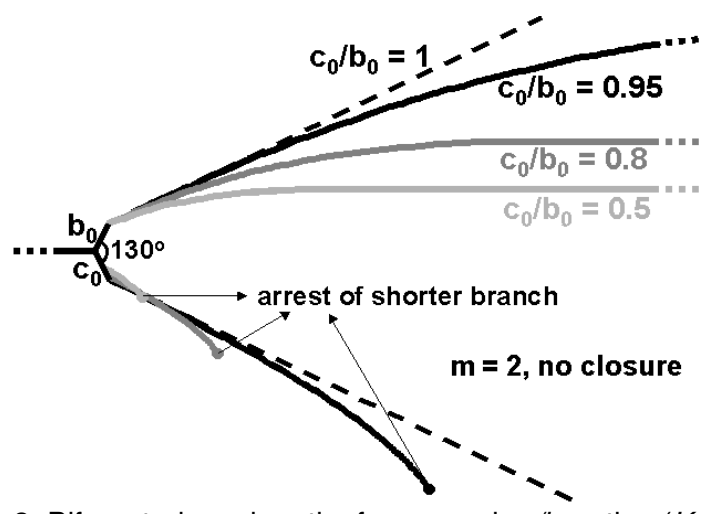

Figure 3. Bifurcated crack paths for several co/bo ratios $\left(K_{P R}=0\right)$.

The size of the retardation zone can be estimated from the ratio $b_{f} / b_{0}$, where $b_{f}$ is the value of the length parameter $b$ of the longer branch (measured along the crack path) beyond which the retardation effect ends. The ratio $b_{f} / b_{0}$ is then calculated through FE propagation simulations for all combinations of $c_{0} / b_{0}=\{0.5,0.8,0.9,0.95\}, 2 \theta=\left\{40^{\circ}, 80^{\circ}, 130^{\circ}, 168^{\circ}\right\}$ and $m=\{2$, $3,4\}$, and fitted by the proposed empirical function:

$$
\frac{b_{f}}{b_{o}}=\exp \left(\frac{2 \theta-30^{o}}{56+17 \cdot(m-2)^{2 / 3}}\right) /\left(1-c_{0} / b_{0}\right)^{(12-m) / 20}
$$

The FE-calculated equivalent SIF $K_{b}$ and $K_{c}$ of the longer and shorter branches are now evaluated along the obtained crack paths. Figure 4 plots the crack retardation factors (defined as the ratios between $K_{b}$ or $K_{c}$ and the Mode I SIF $K_{l}$ of a straight crack) for $2 \theta=130^{\circ}$ and $m=2$, as a function of the normalized length $\left(b-b_{0}\right) / b_{0}$ of the longer branch. Because of the different crack branch lengths, the SIF at the longer one is much higher than that at the shorter branch. Assuming $K_{b}$ and $K_{c}$ to be the crack driving force, it can be seen from Figure 4 that the longer branch reaches its minimum propagation rate right after the 
bifurcation occurs, returning to its pre-overload rate as the crack tip advances away from the influence of the shorter branch. As seen in the figure, the retardation behavior is misleadingly similar to closure-related effects, even though no closure is present in that case.
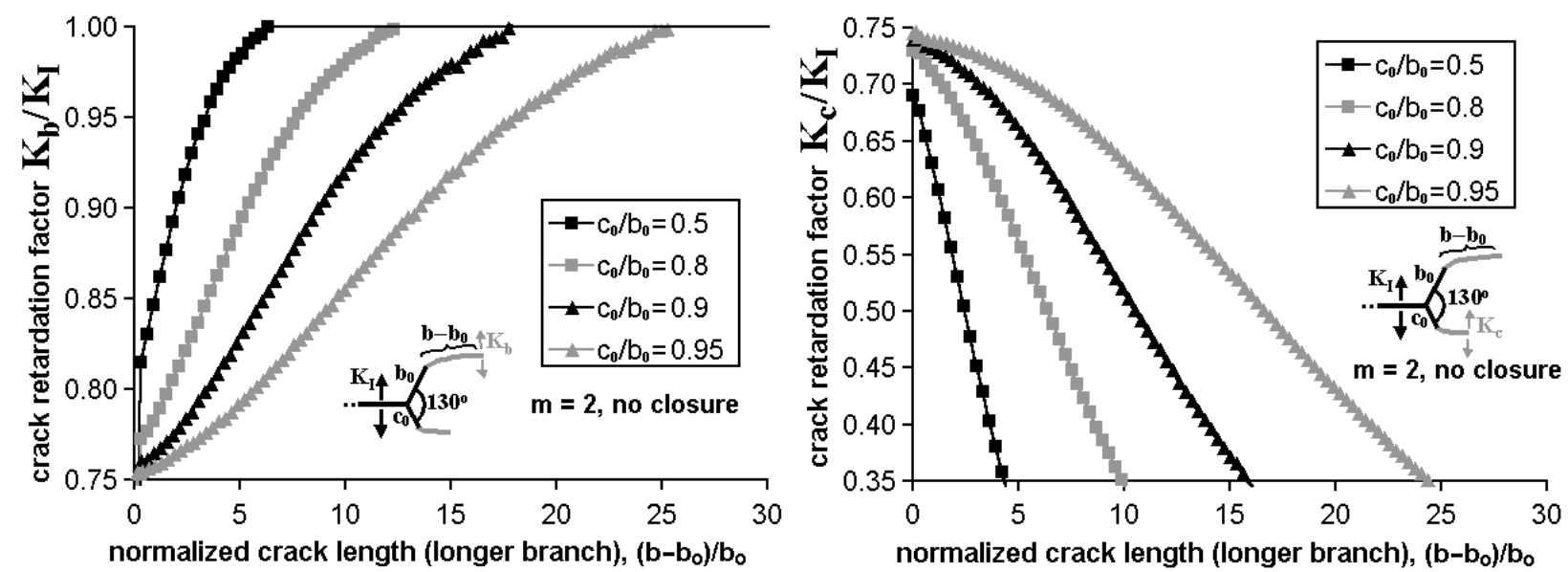

Figure 4. Normalized equivalent SIF for the (a) longer and (b) shorter branch of a bifurcated crack during its propagation $\left(2 \theta=130^{\circ}, m=2, K_{P R}=0\right)$.

In addition, as the length difference between both branches increases, it is expected that the propagation rate of the shorter one is reduced until it arrests, after which the larger branch will dominate. Note that even small differences between the branch lengths, such as in the case $c_{0} / b_{0}=0.95$ shown in Figure 4, are sufficient to cause subsequent arrest of the shorter branch.

An empirical expression is here proposed to model the SIF $K_{b}$ of the longer branch during the transition between $K_{b 0}$ (the value of $K_{b}$ immediately after the bifurcation event) and the straight-crack $K_{l}$ (after the retardation effect ends), valid for $b_{0} \leq b \leq b_{f}$ and $0.7<c_{0} / b_{0}<1$ :

$$
K_{b}=K_{b_{0}}+\left(K_{I}-K_{b_{0}}\right) \cdot\left[a \tan \left(3 \frac{b-b_{0}}{b_{f}-b_{0}}\right) / 1.25\right]^{2 c_{0} / b_{0}}
$$

where $b_{f}$ is given in Equation (4) and $K_{b 0}\left(\right.$ and $K_{c 0}$ ) by

$$
\frac{K_{b 0}}{K_{I}}=0.75+(1-\sin \boldsymbol{\theta}) \cdot\left(1-\frac{c_{0}}{b_{0}}\right), \quad \frac{K_{c 0}}{K_{I}}=0.75-(1-\sin \boldsymbol{\theta}) \cdot\left(1-\frac{c_{0}}{b_{0}}\right)
$$

The interaction between crack branching and other retardation mechanisms is studied next. This effect is easily accounted for in the FE calculations using Equation (3). The limiting value $K_{P R}$ is assumed to be the same at both branch tips and always larger than the minimum SIF of each branch. Further simulations are then conducted considering several $K_{P R}$ values, normalized by the maximum Mode I SIF $K_{l}$ of the straight crack, namely $K_{P R} / K_{I}=\{0.067,0.08,0.10,0.13,0.20,0.25,0.40$, $0.57\}$.

A generalized version of Equation (4) is then proposed to fit the calculated process zone sizes including the combined effects of other mechanisms:

$$
\frac{b_{f}}{b_{o}}=\frac{\boldsymbol{\alpha}}{\left(1-c_{0} / b_{0}\right)^{(12-m) / 20}} \cdot \exp \left[\frac{-\boldsymbol{\beta}}{\left(1-c_{0} / b_{0}\right)^{\boldsymbol{\gamma}}} \cdot \frac{K_{P R}}{K_{I}}\right]
$$

where

$$
\begin{aligned}
& \alpha=\exp \left(\frac{2 \boldsymbol{\theta}-30^{o}}{56+17 \cdot(m-2)^{2 / 3}}\right) \\
& \beta=\left(\frac{2 \boldsymbol{\theta}}{110+60 \cdot(m-2)^{0.6}}\right)^{5 / 2} \\
& \gamma=\frac{180^{o}-2 \boldsymbol{\theta}}{280-130 \cdot(m-2)^{0.3}}
\end{aligned}
$$


Note that the ratio $K_{P R} / K_{l}$ in Equation (7) should be replaced with zero if $K_{P R}$ is smaller than the minimum SIF of both branches. Note also that greater $K_{P R}$ levels result in shorter retardation zones, because the shorter branch is more easily arrested due to the reduction in its stress intensity range.

Figure 5 shows the effect of $K_{P R}$ at the branch tips on the retardation factor for $2 \theta=130^{\circ}, c_{0} / b_{0}=0.9$ and $m=2$. Note that higher $K_{P R}$ levels reduce the size of the retardation process zone, due to premature arrest of the shorter branch. In Fig. 5, e.g., the normalized size of the process zone is reduced from 18 to 3.6 as $K_{P R} / K_{l}$ approaches 0.74 , a factor of 5 . In this example, 0.74 is the minimum $K_{P R} / K_{l}$ level that prevents the shorter branch to even start propagating. Therefore, at any level above 0.74 the normalized process zone size will also be 3.6, because the propagation geometry will remain unchanged as long as the shorter branch remains arrested at $c=c_{0}$.

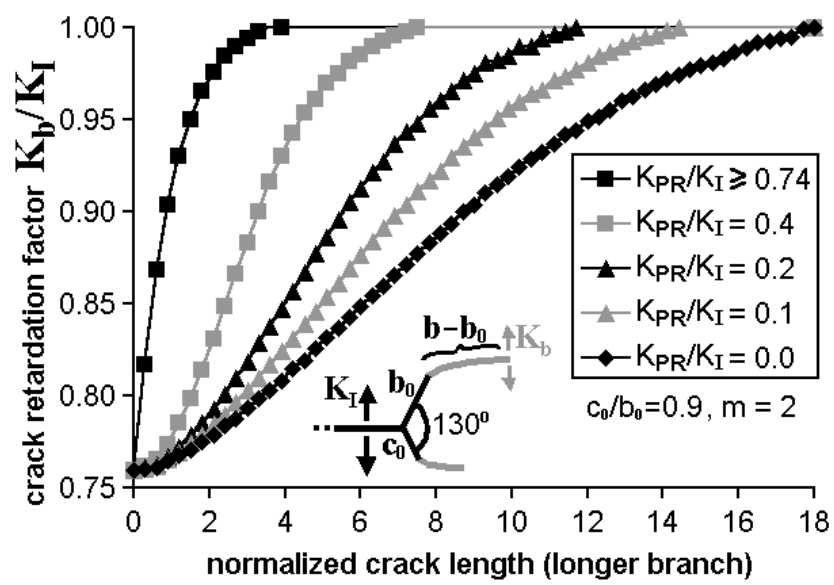

Figure 5. Normalized SIF of the longer branch during its propagation as a function of the normalized length $\left(b-b_{0}\right) / b_{0}$ for several $K_{P R}$ levels $\left(c_{0} / b_{0}=0.9, m=2\right)$.

Note, however, that a smaller process zone does not necessarily mean fewer delay cycles, since the longer branch will also experience a reduction in the crack propagation rate due to other retardation mechanisms. Therefore, a competition between lower growth rates of the longer branch and smaller bifurcation process zone sizes will take place to determine the real effect of combining bifurcation with other retardation mechanisms.

Equations (7-10) and (6) can then be applied to Equation (5) to model the SIF $K_{b}$ of the longer branch during the transition between $K_{b 0}$ (the SIF immediately after the bifurcation event) and the straight-crack $K_{I}$ (the SIF after the end of the retardation effect), completing this analysis.

\section{Experimental Results}

Quantitative validations of the predicted bifurcated crack growth behavior are performed on Eccentrically-loaded Single Edge Crack Tension specimens ESE(T) made from an annealed SAE 4340 alloy steel with $S_{Y}=377 \mathrm{MPa}, S_{U}=660 \mathrm{MPa}, E=$ $205 \mathrm{GPa}$, and $R A=52.7 \%$, and with the analyzed weight percent composition: C 0.37, Mn 0.56, Si 0.14, Ni 1.53, Cr 0.64, Mo 0.18 , S $0.04, \mathrm{P} 0.035$. The tests are performed at frequencies between 20 and $30 \mathrm{~Hz}$ in a $250 \mathrm{kN}$ computer-controlled servohydraulic testing machine. The crack length is measured following ASTM E 647 procedures [17]. Special attention is given for crack closure measurement. For this, a system with a high speed data acquisition is used to obtain data and to avoid intervention during the tests. In this way, the load and Crack-Opening Displacement (COD) data are used to compute the crack closure load using a digital linearity subtractor output (a circuit developed to precisely measure the opening load, [18]).

The proposed retardation equations are implemented in a fatigue life assessment program named ViDa. This program is used to estimate the number of delay cycles associated with the experimentally obtained bifurcation on the $4340 \mathrm{steel} E S E(T)$ specimen. The number of cycles spent during the propagation in the retardation region is then calculated by integrating the $d a / d N$ equation along the longer crack branch, from $b=b_{0}$ to $b=b_{f}$.

Two tests are performed on $\mathrm{ESE}(\mathrm{T})$ specimens subject to $100 \%$ overloads, namely tests I and II: (I) $R=0.7, \Delta K=13.9$ $\mathrm{MPa} \sqrt{\mathrm{m}}$, resulting in $\approx 22,000$ delay cycles; and (II) $R=0.7, \Delta K=14.2 \mathrm{MPa} \sqrt{\mathrm{m}}$, resulting in approximately 20,000 delay cycles, see Figs. 6-7. 


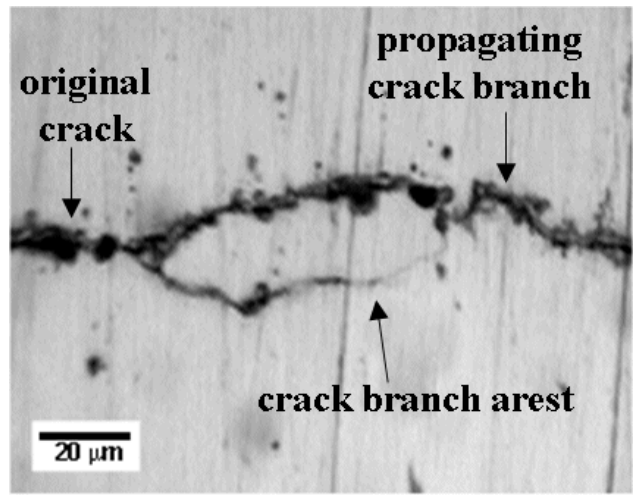

(a)

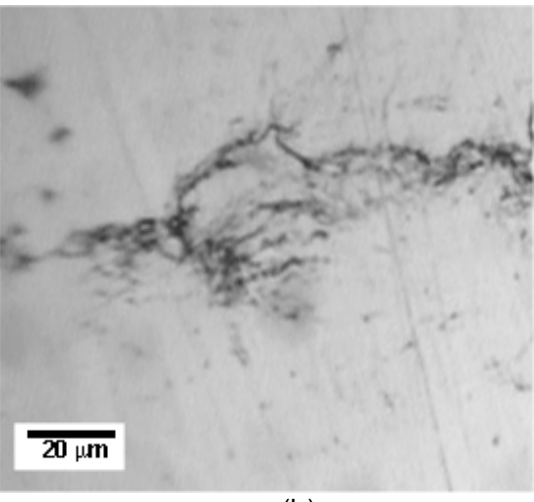

(b)

Fig. 6. Crack bifurcation experiments on SAE 4340 steel: (a) test I, (b) test II.
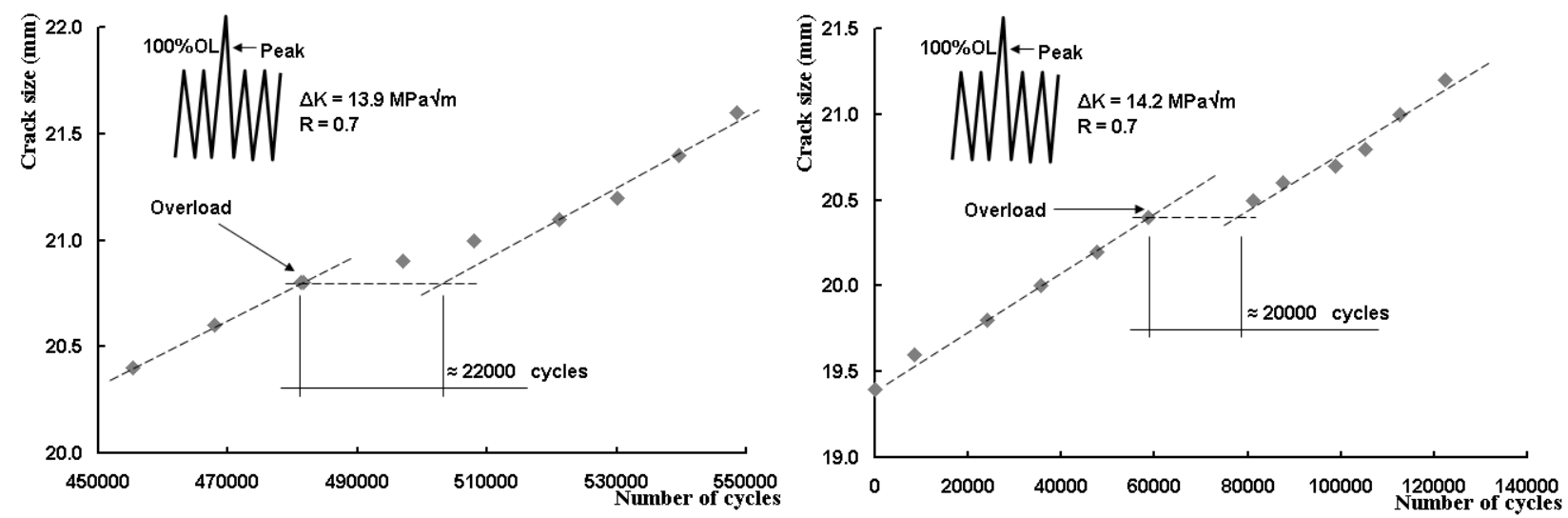

Fig. 7. Fatigue crack growth retardation after a $100 \%$ overload, $\mathrm{R}=0.7$ : (a) test I, (b) test II.

It is found that the minimum load levels in tests I and II are always above the opening load, therefore no crack closure is present nor before nor after the overloads. For test I, the measured initial branch lengths are approximately $b_{0}=9 \mu \mathrm{m}$ and $c_{0}=$ $8.5 \mu \mathrm{m}$, with a bifurcation angle $2 \theta=160^{\circ}$, see Fig. 6(a). The material is modeled using Equation (1) with crack growth constants $A=9 \cdot 10^{-11} \mathrm{~m} /$ cycle and $m=2.1$, and a propagation threshold $\Delta K_{t h}=2.8 \mathrm{MPa} \sqrt{\mathrm{m}}$, measured under $R=0.7$. From Equation (6), it is found that $K_{b 0} / K_{l}=0.751$ and $K_{c o} / K_{l}=0.749$, leading to $\Delta K_{b 0}=0.751 \cdot \Delta K_{l}=10.437 \mathrm{MPa} \sqrt{\mathrm{m}}$ and $\Delta K_{c 0}=$ $0.749 \cdot \Delta K_{l}=10.413 \mathrm{MPa} \sqrt{\mathrm{m}}$. Since both ranges are greater than $\Delta K_{t h}(R=0.7)=2.8 \mathrm{MPa} \sqrt{\mathrm{m}}$, both branches are expected to start propagating, as verified experimentally. The size of the process zone can be estimated from Equation (4), which results in $b_{f}=$ $36.95 \times 9 \mu \mathrm{m} \cong 332 \mu \mathrm{m}$. The number of delay cycles $n_{D}$ is then calculated by:

$$
\begin{aligned}
& n_{D}=\int_{b_{0}}^{b_{f}} \frac{d b}{A\left(\Delta K_{b}-\Delta K_{t h}\right)^{m}}-\int_{b_{0}}^{b_{f}} \frac{d b}{A\left(\Delta K_{I}-\Delta K_{t h}\right)^{m}}= \\
& =\int_{9}^{332} \frac{d b \times 10^{-6}}{9 \cdot 10^{-11}\left\{7.64+2.27 \cdot\left[a \tan \left(3 \frac{b-9}{332-9}\right) / 1.25\right]^{1.88}\right\}^{2.1}}-\int_{9}^{332} \frac{d b \times 10^{-6}}{9 \cdot 10^{-11}(13.9-3.8)^{2.1}}=9,664 \text { cycles }
\end{aligned}
$$

which is approximately half of the measured 22,000 delay cycles, see Fig. 7(a).

For test II, the measured initial branch lengths are approximately $b_{0}=10 \mu \mathrm{m}$ and $c_{0}=9.5 \mu \mathrm{m}$, but with a larger bifurcation angle $2 \theta=160^{\circ}$, see Fig. $6($ b) , resulting in:

$$
n_{D}=\int_{b_{0}}^{b_{f}} \frac{d b}{A\left(\Delta K_{b}-\Delta K_{t h}\right)^{m}}-\int_{b_{0}}^{b_{f}} \frac{d b}{A\left(\Delta K_{I}-\Delta K_{t h}\right)^{m}}=36,093-25,424=10,669
$$

which is also about half of the measured 20,000 delay cycles, see Fig. 7 (b). 
Note that in all tests there was no retardation induced by crack closure. The opening load remained lower than before the overload along the entire process zone, only returning to its original value after the bifurcation effect had ended. Therefore closure would not be able to explain the measured overload-induced retardation. This behavior has already been verified in the literature [19].

The errors in the predictions performed using the proposed semi-empirical equations can be explained by inaccuracies in the estimation of the initial branch lengths $b_{0}$ and $c_{0}$, since the retardation effect is highly dependent on the ratio $c_{0} / b_{0}$. In addition, other retardation mechanisms besides bifurcation (except for closure, as discussed above) might be contributing to increase the number of delay cycles, such as unmodeled environmental effects or further kinking of the branch tips due to microstructure inhomogeneities. Even so, the presented predictions are of the same order of magnitude of the experimental scatter. Therefore, the quantitative approach presented in this work is a quite promising tool for modeling and calculating overload-induced retardation effects where other mechanisms have failed to give a satisfactory explanation.

Finally, it must be noted as well that all measured bifurcations occurred throughout the thickness in an approximately uniform pattern, observed after carefully slicing and reexamining the specimens. Therefore, despite the inherent 3-D nature of the bifurcation problem, in these tests the presented two-dimensional FE approach has been validated.

\section{Conclusions}

In this work, a specialized FE program was used to calculate the propagation path and associated stress intensity factors (SIF) of bifurcated cracks, which can cause crack retardation or even arrest. A total of 262 crack propagation simulations were obtained from a total of $6,250 \mathrm{FE}$ calculation steps to fit empirical equations to the process zone size and crack retardation factor along the curved crack branches. In particular, the bifurcation simulations included several combinations of bifurcation angles $2 \theta=\left\{40^{\circ}, 80^{\circ}, 90^{\circ}, 130^{\circ}, 168^{\circ}\right\}$, branch asymmetry ratios $c_{0} / b_{0}=\{0.5,0.7,0.8,0.9,0.95,1.0\}$, crack growth exponents $m=\{2,3,4\}$, and even considered interaction between crack branching and other retardation mechanisms through the threshold ratios $K_{P R} / K_{I}=\{0.0,0.067,0.08,0.10,0.13,0.20,0.25,0.40,0.57\}$. It was shown that very small differences between the lengths of the bifurcated branches are sufficient to cause the shorter one to eventually arrest as the longer branch returns to the pre-overload conditions. The process zone size was found to be smaller for lower bifurcation angles and for branches with greater asymmetry, in both cases due to the increased shielding effects on the shorter branch. The retardation zone was reduced as well for materials with higher crack growth exponents, due to the increased difference between the crack growth rates of the longer and shorter branches. Higher $K_{P R}$ levels also resulted in smaller process zones, because the shorter branch was more easily arrested due to the reduction in its stress intensity range. However, a competition between smaller process zone sizes and lower growth rates of the longer branch did take place to determine the real effect of combined bifurcation and other retardation mechanisms. The proposed equations, besides capturing all above described phenomena, can be readily used to predict the propagation behavior of branched and kinked cracks in an arbitrary structure, as long as the process zone is small compared to the other characteristic dimensions. It should be recognized however that the presented mixed-mode equations are only accurate if the branch lengths greatly exceed the size scale of the microstructural inhomogeneities and the size of the near-tip plastic zone. But assuming that the entire crack-front bifurcates uniformly, as observed in this work from scanning electron micrographs, the specimen thickness itself may provide the size scale requirements for the validity of the presented equations, as the calculated SIF may be averaged considering the (several) grains present along the thickness. Otherwise, if the crack deflections vary significantly along the thickness, then further modeling including Mode III effects should be considered. From these results, it can be seen that crack bifurcation may provide an alternate mechanistic explanation for overload-induced crack retardation on structural components, in special to explain load interaction effects under closure-free conditions.

\section{References}

1. Lankford J, Davidson DL. The Effect of Overloads upon Fatigue Crack Tip Opening Displacement and Crack Tip Opening/Closing Loads in Aluminum Alloys. Advances In Fracture Research, Pergamon Press, 2, 899-906 (1981).

2. Erdogan, F., Sih, G.C. On the Crack Extension in Plates under Plane Loading and Transverse Shear. Journal of Basic Engineering, 85, 519-527 (1963).

3. Suresh S. Crack Deflection: Implications for the Growth of Long and Short Fatigue Cracks. Metallurgical Transactions, 14a, 2375-85 (1983).

4. Kosec B, Kovacic G, Kosec L. Fatigue Cracking of an Aircraft Wheel. Engineering Failure Analysis, 9, 603-9 (2002).

5. Karihaloo BL. On Crack Kinking and Curving. Mechanics of Materials, 1, 189-201 (1982).

6. Seelig T, Gross D. On the Interaction and Branching of Fast Running Cracks - A Numerical Investigation. Journal of the Mechanics and Physics of Solids, 47, 935-52 (1999).

7. Suresh S. Micromechanisms of Fatigue Crack Growth Retardation Following Overloads. Engineering Fracture Mechanics, 18(3), 577-93 (1983).

8. Suresh S. Fatigue of Materials. Cambridge University Press, 679p (1988).

9. Melin S. Why do cracks avoid each other? International Journal of Fracture, 23, 37-45 (1983). 
10. Pärletun LG. Determination of the growth of branched cracks by numerical methods. Engineering Fracture Mechanics, 11, 343-358 (1979).

11. Suresh S, Shih CF. Plastic Near-tip Fields for Branched Cracks. International Journal of Fracture, 30, $237-259$ (1986).

12. Miranda ACO, Meggiolaro MA, Castro JTP, Martha LF, Bittencourt TN. Fatigue Crack Propagation under Complex Loading in Arbitrary 2D Geometries, ASTM STP 1411, 4, 120-46 (2002).

13. Miranda ACO, Meggiolaro MA, Castro JTP, Martha LF, Bittencourt TN. Fatigue Life and Crack Path Prediction in Generic 2D Structural Components. Eng. Fracture Mechanics, 70, 1259-79 (2003).

14. Lang M, Marci G. The influence of single and multiple overloads on fatigue crack propagation. Fatigue and Fracture of Engineering Materials and Structures, 22, 257-271 (1999).

15. Sadananda K, Vasudevan AK, Holtz RL. Extension of the Unified Approach to Fatigue Crack Growth to Environmental Interactions. International Journal of Fatigue, 23, S277-S286 (2001).

16. Sadananda K, Vasudevan AK. Fatigue Crack Growth Mechanisms in Steels. International Journal of Fatigue, 25, 899-914 (2003).

17. ASTM Standard E 647. Standard Test Method for Measurement of Fatigue Crack Growth Rates. ASTM Standards;03.01

18. Castro JTP. A Circuit to Measure Crack Closure, Exp. Techniques, 17(2), 23-25 (1993).

19. Meggiolaro MA, Castro JTP. On the dominant role of crack closure on fatigue crack growth modeling. International Journal of Fatigue, 25(9-11), 843-854 (2003). 